\title{
Bäcklund transformations for higher order Painlevé equations
}

\author{
P.R. Gordoa ${ }^{\text {a,* }}$, U. Muğan ${ }^{\text {b }}$, A. Pickering ${ }^{\text {c }}$, A. Sakka ${ }^{\text {d }}$ \\ ${ }^{a}$ Fac. de Ciencias, Area de Fisica Teórica, Universidad de Salamanca, 37008 Salamanca, Spain \\ ${ }^{\mathrm{b}}$ Department of Mathematics, Bilkent University, 06800 Bilkent, Ankara, Turkey \\ c Departamento de Matemáticas, Universidad de Salamanca, Plaza de la Merced 1, 37008 Salamanca, Spain \\ ${ }^{\mathrm{d}}$ Department of Mathematics, Islamic University of Gaza, P.O. Box 108, Rimal, Gaza, Palestine
}

Accepted 17 February 2004

Communicated by Prof. M. Wadati

\begin{abstract}
We present a new generalized algorithm which allows the construction of Bäcklund transformations (BTs) for higher order ordinary differential equations (ODEs). This algorithm is based on the idea of seeking transformations that preserve the Painlevé property, and is applied here to ODEs of various orders in order to recover, amongst others, their auto-BTs. Of the ODEs considered here, one is seen to be of particular interest because it allows us to show that autoBTs can be obtained in various ways, i.e. not only by using the severest of the possible restrictions of our algorithm. (C) 2004 Elsevier Ltd. All rights reserved.
\end{abstract}

\section{Introduction}

One area of research that for some time now has attracted considerable interest is the study of properties of the six Painlevé equations $P_{\mathrm{I}}, \ldots, P_{\mathrm{VI}}[1-4]$. One property that is generally considered to be of particular importance is the existence of Bäcklund transformations (BTs), that is, transformations relating a particular Painlevé equation either to itself (with possibly different values of the parameters appearing as coefficients), or to another equation with the Painlevé property. Various approaches to the recovery of BTs can be found for example in [5-14]; see [8] for a list of references prior to 1980 .

The Painlevé equations, as is well known, resulted from the classification of second order ordinary differential equations (ODEs), within a certain class, having what today is referred to as the Painlevé property. This classification was motivated by the search for new transcendental functions. The work of Painlevé was extended to higher order ODEs by authors such as Chazy [15], Garnier [16], Bureau [17], Exton [18] and Martynov [19], although no complete classification has yet been given for a class of ODEs as general as that originally considered at second order. More recent work that has continued this classical approach to Painlevé classification can be found in [20-25]. An alternative approach to the problem of obtaining new integrable ODEs, based on the use of non-isospectral scattering problems, can be found in [26-29]. It is amongst the equations found in [20-29] that ODEs defining new transcendental functions might be expected to be found. This then leads naturally to the problem of studying the properties of such new ODEs.

For the Painlevé equations, the study of BTs has been undertaken by a great number of different authors (see the references given above). One well-known approach is that adopted in [8]. In this approach, an ansatz is made relating

\footnotetext{
${ }^{*}$ Corresponding author. Present address: Area de Matemática Aplicada, ESCET, Universidad Rey Juan Carlos, C/Tulipan s/n, 28933 Móstoles, Madrid, Spain. Fax: +34-914-887-338.

E-mail address: mpruiz@escet.urjc.es (P.R. Gordoa).
} 
the solutions of a Painlevé equation in $v(z)$ to those of a second order ODE in $u(z)$ having the Painlevé property; the ansatz used in [8] relates $v(z)$ and $u(z)$ via

$$
\left(d v^{2}+e v+f\right) u-\left(v^{\prime}+a v^{2}+b v+c\right)=0,
$$

where $a, b, c, d, e$ and $f$ are all functions of $z$ only. The algorithm presented in [8] then determines the precise forms of both the BT (1) and the ODE in $u(z)$, this last by construction in [8] being at most quadratic in $u^{\prime \prime}(z)$.

Various generalizations of this approach have since appeared in the literature. In $[30,31]$ the same ansatz (1) was used to obtain second order second degree ODEs related to $P_{\mathrm{I}}, \ldots, P_{\mathrm{VI}}$. In [32], instead of the ansatz (1), the ansatz

$$
\left[\left(\sum_{i=0}^{2} c_{i} v^{i}\right) v^{\prime}+\sum_{i=0}^{4} d_{i} v^{i}\right] u-\left[\left(v^{\prime}\right)^{2}+\left(\sum_{i=0}^{2} a_{i} v^{i}\right) v^{\prime}+\sum_{i=0}^{4} b_{i} v^{i}\right]=0,
$$

where all $a_{i}, b_{i}, c_{i}$ and $d_{i}$ are functions of $z$ only, was used to find further second order second degree ODEs related to $P_{\mathrm{I}}, \ldots, P_{\mathrm{VI}}$. In [33] (2) was used to obtain second order fourth degree ODEs related to $P_{\mathrm{I}}, \ldots, P_{\mathrm{IV}}$.

Meanwhile in [34] it was noted, using as examples $P_{\mathrm{III}}$ and $P_{\mathrm{IV}}$, that the ansatz (1) can be used to obtain BTs to ODEs of degree higher than two. In [35] a generalized version of the algorithm in [8] was given, allowing the construction of BTs for $n$th order ODEs, in quite a general class, to ODEs of the same order but perhaps of higher degree; as an example this generalized approach was applied to a particular fourth order ODE believed to define a new transcendental function. This generalized algorithm has also been applied in [36] to the fourth order analogue of $P_{\mathrm{I}}$, and in [37] to the generalized fourth order analogue of $P_{\mathrm{II}}$. In [38] the approach developed in [34,35] was applied to $P_{\mathrm{I}}$ and $P_{\mathrm{II}}$ to obtain BTs to second order ODEs of degree greater than two. We note that an alternative approach to finding BTs appears in [39-41].

The aim of the present paper is to consider further generalizations of the above approaches, in order to obtain BTs for higher order Painlevé equations. We begin in Section 2 by seeking BTs for fourth order ODEs using an ansatz first suggested, though not actually used, in [35]. It is analogues of this ansatz that we use to seek BTs in subsequent sections. We give here a general description of this approach. Further generalizations are of course always possible (see [35] for a discussion).

We consider $n$th order equations with the Painlevé property of the form

$$
v^{(n)}=f\left(z, v, v^{\prime}, \ldots, v^{(n-2)}, v^{(n-1)}\right),
$$

where $f$ is a rational function of $v^{(n-1)}, \ldots, v$ with coefficients functions of $z$. Here we consider, instead of (1), a transformation of the form

$$
G\left(z, v, \ldots, v^{(n-2)}\right) u-\left[v^{(n-1)}+F\left(z, v, \ldots, v^{(n-2)}\right)\right]=0,
$$

where the functions $F$ and $G$ guarantee that the equation in $u$ also has the Painlevé property, i.e. that (4) is a transformation that preserves the Painlevé property. In the current paper we will take both $F$ and $G$ to be polynomial in $v^{(n-2)}, \ldots, v$, with coefficients functions of $z$ only, such that each monomial is of maximum weight $n$ under the rescaling $(v, \mathrm{~d} / \mathrm{d} z) \rightarrow(\lambda v, \lambda \mathrm{d} / \mathrm{d} z)$.

Differentiating (4) once and replacing $v^{(n)}$ using (3), and then $v^{(n-1)}$ using (4), yields a polynomial in $v, v^{\prime}, \ldots, v^{(n-2)}, u, u^{\prime}$ with coefficients functions of $z$. Elimination of $v$ between this last and (4) then yields the $n$th order ODE satisfied by $u$. This elimination process can be simplified by insisting on special choices of the coefficients in the BT (4) such that the polynomial in $v, v^{\prime}, \ldots, v^{(n-2)}, u, u^{\prime}$ reduces to a polynomial in $v, v^{\prime}, \ldots, v^{(p)}, u, u^{\prime}$ for some $p<n-2$; in particular we might ask that it reduces to a polynomial in $v, u, u^{\prime}$. In the special case $n=2$, which is that considered in [8], this polynomial will already be a polynomial in $v, u, u^{\prime}$ only.

The aim of the present paper is to show how the above algorithm can be used to derive BTs, and in particular autoBTs, for higher order Painlevé equations. In Section 2 we apply this algorithm to two fourth order ODEs widely believed to define new transcendents; in Section 3 we consider two sixth order ODEs, higher order analogues of those in Section 2. We limit ourselves in Sections 2 and 3 to the case where we reduce the polynomial whose derivation is outlined above to one in $v, u, u^{\prime}$ only. The reason for this is that this is enough to allow us to recover auto-BTs for the ODEs considered. It is in Section 4 that we consider whether auto-BTs can be recovered without reducing this polynomial to one in $v, u, u^{\prime}$. Thus we consider the application of our algorithm to a third order ODE; we find that, in this 
case, a less severe restriction, to a polynomial in $v, v^{\prime}, u, u^{\prime}$ but linear in $v^{\prime}$, can also be used in order to recover an autoBT. Section 5 is devoted to conclusions, to a consideration of possible generalizations of our approach, and also to a discussion of the mathematical foundations underlying this kind of approach to deriving BTs.

\section{Fourth order Painlevé equations}

We consider in this section the application of the algorithm outlined above to two fourth order Painlevé equations. Thus we seek a BT of the form

$$
G\left(z, v, v^{\prime}, v^{\prime \prime}\right) u-\left[v^{\prime \prime \prime}+F\left(z, v, v^{\prime}, v^{\prime \prime}\right)\right]=0
$$

where $F$ and $G$ are given by

$$
\begin{aligned}
& F=-\left(a_{01} v+a_{00}\right) v^{\prime \prime}-b_{00}\left(v^{\prime}\right)^{2}-\left(c_{02} v^{2}+c_{01} v+c_{00}\right) v^{\prime}-\left(d_{04} v^{4}+d_{03} v^{3}+d_{02} v^{2}+d_{01} v+d_{00}\right), \\
& G=\left(a_{11} v+a_{10}\right) v^{\prime \prime}+b_{10}\left(v^{\prime}\right)^{2}+\left(c_{12} v^{2}+c_{11} v+c_{10}\right) v^{\prime}+\left(d_{14} v^{4}+d_{13} v^{3}+d_{12} v^{2}+d_{11} v+d_{10}\right)
\end{aligned}
$$

and where all $a_{i j}, b_{i 0}, c_{i j}$, and $d_{i j}$ are functions of $z$ only. In order to simplify the presentation of our results we rewrite the BT (5) as

$$
v^{\prime \prime \prime}=\left(A_{1} v+A_{0}\right) v^{\prime \prime}+B_{0}\left(v^{\prime}\right)^{2}+\left[C_{2} v^{2}+C_{1} v+C_{0}\right] v^{\prime}+D_{4} v^{4}+D_{3} v^{3}+D_{2} v^{2}+D_{1} v+D_{0}
$$

where

$$
\begin{aligned}
& A_{j}=a_{1 j} u+a_{0 j}, \quad j=0,1, \\
& B_{0}=b_{10} u+b_{00}, \\
& C_{j}=c_{1 j} u+c_{0 j}, \quad j=0,1,2, \\
& D_{j}=d_{1 j} u+d_{0 j}, \quad j=0,1,2,3,4 .
\end{aligned}
$$

The two fourth order Painlevé equations dealt with in this section have the form

$$
v^{(4)}=\left[P_{1}(z, v) v^{\prime}+P_{0}(z, v)\right] v^{\prime \prime}+Q_{2}(z, v)\left(v^{\prime}\right)^{2}+Q_{1}(z, v) v^{\prime}+Q_{0}(z, v),
$$

where all $P_{i}$ and $Q_{i}$ are polynomial in $v$ with coefficients functions of $z$. We assume that the BT to an ODE in $u$ is as given by (5), and follow the procedure outlined in Section 1. Differentiating Eq. (5) and using Eq. (10) to replace $v^{(4)}$, and Eq. (5) to replace $v^{\prime \prime \prime}$, yields the relation

$$
\left(\psi_{1} v^{\prime}+\psi_{0}\right) v^{\prime \prime}+\phi_{2}\left(v^{\prime}\right)^{2}+\phi_{1} v^{\prime}+\phi_{0}=0
$$

where all $\psi_{i}$ and $\phi_{i}$ are polynomials in $v, u, u^{\prime}$ with coefficients functions of $z$. Elimination of $v$ between this relation and (5) leads to an ODE in $u$.

In this section we consider the following simplification of this elimination procedure: we choose $A_{i}, B_{0}, C_{i}$, and $D_{i}$ (i.e. $a_{i j}, b_{i 0}, c_{i j}$ and $d_{i j}$ ) so that $\psi_{j}, j=0,1$ and $\phi_{j}, j=1,2$ are identically zero. In this case Eq. (11) reduces to a polynomial in $v$ (with coefficients polynomials in $u, u^{\prime}$ with coefficients functions of $z$ ),

$$
\phi_{0}(v, z)=0
$$

which then defines the inverse of the transformation (5). Elimination of $v$ between this last equation and (5), in order to find the ODE in $u$, is thus made much easier. We now turn to our examples.

\subsection{Example 2.1}

As our first example we consider the equation

$$
v^{(4)}=10 v^{2} v^{\prime \prime}+10 v\left(v^{\prime}\right)^{2}-6 v^{5}-\beta\left(v^{\prime \prime}-2 v^{3}\right)+z v+\alpha,
$$

which is the second member of the generalized second Painlevé or $P_{\mathrm{II}}$ hierarchy; see [5] for the $P_{\mathrm{II}}$ hierarchy, [21,25] for the above fourth order ODE, and [42] for the generalized $P_{\mathrm{II}}$ hierarchy. We find that $\psi_{i}$ and $\phi_{i}$ in (11) are given by 


$$
\begin{aligned}
\psi_{1}= & A_{1}+2 B_{0}, \\
\psi_{0}= & \left(A_{1}^{2}+C_{2}-10\right) v^{2}+\left(2 A_{1} A_{0}+A_{1}^{\prime}+C_{1}\right) v+A_{0}^{2}+A_{0}^{\prime}+C_{0}+\beta, \\
\phi_{2}= & \left(A_{1} B_{0}+2 C_{2}-10\right) v+A_{0} B_{0}+B_{0}^{\prime}+C_{1}, \\
\phi_{1}= & \left(A_{1} C_{2}+4 D_{4}\right) v^{3}+\left(A_{1} C_{1}+A_{0} C_{2}+C_{2}^{\prime}+3 D_{3}\right) v^{2} \\
& +\left(A_{1} C_{0}+A_{0} C_{1}+C_{1}^{\prime}+2 D_{2}\right) v+A_{0} C_{0}+C_{0}^{\prime}+D_{1}, \\
\phi_{0}= & \left(A_{1} D_{4}+6\right) v^{5}+\left(D_{4}^{\prime}+A_{1} D_{3}+A_{0} D_{4}\right) v^{4} \\
& +\left(D_{3}+A_{1} D_{2}+A_{0} D_{3}-2 \beta\right) v^{3}+\left(D_{2}^{\prime}+A_{1} D_{1}+A_{0} D_{2}\right) v^{2} \\
& +\left(D_{1}^{\prime}+A_{1} D_{0}+A_{0} D_{1}-z\right) v+D_{0}^{\prime}+A_{0} D_{0}-\alpha .
\end{aligned}
$$

Imposing that $\psi_{j}, j=0,1$ and $\phi_{j}, j=1,2$ be identically zero, implies that $A_{0}=C_{1}=D_{1}=D_{3}=0, A_{1}=-2 \epsilon, B_{0}=\epsilon$, $C_{2}=6, C_{0}=-\beta, D_{4}=3 \epsilon$ and $D_{2}=-\epsilon \beta$, where $\epsilon= \pm 1$. Without loss of generality we may also set $D_{0}=u$. The resulting equation $\phi_{0}=0$ (12) then reads

$$
(2 \epsilon u+z) v-\left(u^{\prime}-\alpha\right)=0
$$

and the transformation (5) becomes

$$
u=v^{\prime \prime \prime}+2 \epsilon v v^{\prime \prime}-\epsilon\left(v^{\prime}\right)^{2}-\left(6 v^{2}-\beta\right) v^{\prime}-3 \epsilon v^{4}+\epsilon \beta v^{2} .
$$

We now introduce, for reasons that will become clear shortly, the new variable $y(x)=\frac{2 \epsilon}{2 \epsilon u+z}$. Eq. (15) then becomes

$$
v=\frac{-\epsilon\left(y^{\prime}+v y^{2}\right)}{2 y}
$$

where $v=\alpha+\frac{\epsilon}{2}$, and Eq. (16) becomes

$$
y=\frac{2}{2 v^{\prime \prime \prime}+4 \epsilon v v^{\prime \prime}-2 \epsilon\left(v^{\prime}\right)^{2}-2\left(6 v^{2}-\beta\right) v^{\prime}-6 \epsilon v^{4}+2 \epsilon \beta v^{2}+\epsilon z} .
$$

Substituting $v$ from (17) into (18) then yields the fourth order ODE in $y$,

$$
y^{(4)}=\frac{5 y^{\prime} y^{\prime \prime \prime}}{y}+\frac{5\left(y^{\prime \prime}\right)^{2}}{2 y}-\left[\frac{25\left(y^{\prime}\right)^{2}}{2 y^{2}}-\frac{5}{2} v^{2} y^{2}+\beta\right] y^{\prime \prime}+\frac{45\left(y^{\prime}\right)^{4}}{8 y^{3}}-\left[\frac{5}{4} v^{2} y^{2}-\frac{3}{2} \beta\right] \frac{\left(y^{\prime}\right)^{2}}{y}-\frac{3}{8} v^{4} y^{5}+\frac{1}{2} \beta v^{2} y^{3}+z y-2 \epsilon .
$$

Thus we obtain the BT (17), (18) between the second member of the generalized $P_{\mathrm{II}}$ hierarchy (13), and Eq. (19). We now use this result to derive auto-BTs for Eq. (13).

\subsubsection{Auto-Bäcklund transformations for Eq. (13)}

We now use the BT obtained above to derive auto-BTs for Eq. (13). Here we make use of the fact that Eq. (19) is invariant under $v \rightarrow-v$.

We begin by noting that the BT (17), (18) defines a mapping between solutions $v$ of (13) for parameter value $\alpha=v-\frac{\epsilon}{2}$ and solutions $y$ of (19) for parameter value $v$. Changing the sign of $v$ in this BT then yields an alternative BT consisting of the two relations

$$
v=\frac{-\epsilon\left(y^{\prime}-v y^{2}\right)}{2 y}
$$

and (18), between solutions $v$ of (13) for parameter value $\alpha=-v-\frac{\epsilon}{2}$ and solutions $y$ of (19).

Thus given a solution $y$ of (19) we can obtain two solutions $v$ and $\bar{v}$ of (13),

$$
\begin{aligned}
& v=\frac{-\epsilon\left(y^{\prime}+v y^{2}\right)}{2 y}, \\
& \bar{v}=\frac{-\epsilon\left(y^{\prime}-v y^{2}\right)}{2 y},
\end{aligned}
$$

for parameter values $\alpha=v-\frac{\epsilon}{2}$ and $\bar{\alpha}=-v-\frac{\epsilon}{2}$ respectively. Since we are using the same solution $y$ to obtain $v$ and $\bar{v}$, subtracting (21) and (22) gives 


$$
\bar{v}=v+\epsilon v y .
$$

Substituting for $y$ from (18) and noting that $\alpha+\bar{\alpha}=-\epsilon$ yields the auto-BT

$$
\begin{aligned}
& \bar{v}=v+\frac{2 \epsilon \alpha+1}{2 v^{\prime \prime \prime}+4 \epsilon v v^{\prime \prime}-2 \epsilon\left(v^{\prime}\right)^{2}-2\left(6 v^{2}-\beta\right) v^{\prime}-6 \epsilon v^{4}+2 \epsilon \beta v^{2}+\epsilon z}, \\
& \bar{\alpha}=-(\alpha+\epsilon),
\end{aligned}
$$

which relates two solutions $v$ and $\bar{v}$ of the same Eq. (13) for parameter values $\alpha$ and $\bar{\alpha}$ respectively. This BT is a generalization of the BT first obtained by Airault [5] for the second member of the $P_{\mathrm{II}}$ hierarchy; we note that the above result for (13) was also obtained in [37] using the different ansatz (1). Thus we see that our approach allows us to derive auto-BTs for Eq. (13).

\subsection{Example 2.2}

As a second example of the application of our method we consider the equation

$$
v^{(4)}=-5 v^{\prime} v^{\prime \prime}+5 v^{2} v^{\prime \prime}+5 v\left(v^{\prime}\right)^{2}-v^{5}+z v-\frac{1}{2} \alpha
$$

which has recently been proposed as defining a new transcendent [21,25]. Proceeding as in our previous example, we find this time that the coefficients $\psi_{i}$ and $\phi_{i}$ in (11) are given by

$$
\begin{aligned}
\psi_{1}= & A_{1}+2 B_{0}+5 \\
\psi_{0}= & \left(A_{1}^{2}+C_{2}-5\right) v^{2}+\left(2 A_{1} A_{0}+A_{1}^{\prime}+C_{1}\right) v+A_{0}^{2}+A_{0}^{\prime}+C_{0} \\
\phi_{2}= & \left(A_{1} B_{0}+2 C_{2}-5\right) v+A_{0} B_{0}+B_{0}^{\prime}+C_{1} \\
\phi_{1}= & \left(A_{1} C_{2}+4 D_{4}\right) v^{3}+\left(A_{1} C_{1}+A_{0} C_{2}+C_{2}^{\prime}+3 D_{3}\right) v^{2} \\
& +\left(A_{1} C_{0}+A_{0} C_{1}+C_{1}^{\prime}+2 D_{2}\right) v+A_{0} C_{0}+C_{0}^{\prime}+D_{1} \\
\phi_{0}= & \left(A_{1} D_{4}+1\right) v^{5}+\left(D_{4}^{\prime}+A_{1} D_{3}+A_{0} D_{4}\right) v^{4}+\left(D_{3}^{\prime}+A_{1} D_{2}+A_{0} D_{3}\right) v^{3} \\
& +\left(D_{2}^{\prime}+A_{1} D_{1}+A_{0} D_{2}\right) v^{2}+\left(D_{1}^{\prime}+A_{1} D_{0}+A_{0} D_{1}-z\right) v+D_{0}^{\prime}+A_{0} D_{0}+\frac{1}{2} \alpha .
\end{aligned}
$$

In order to make $\psi_{j}, j=0,1$ and $\phi_{j}, j=1,2$ identically zero, we have to choose $A_{0}=C_{0}=C_{1}=D_{1}=D_{2}=D_{3}=0$, $A_{1}=-\left(2 B_{0}+5\right), C_{2}=5-A_{1}^{2}$ and $D_{4}=-\frac{1}{4} A_{1} C_{2}$. Also we have two possible values of $B_{0}$, namely $B_{0}=-3$ or $B_{0}=-\frac{3}{2}$, and, without loss of generality, we may set $D_{0}=u$. We will now consider these two cases separately.

Case 1: $B_{0}=-3$. In this case, we have $A_{1}=1, C_{2}=4, D_{4}=-1$ and the equation $\phi_{0}=0$ becomes

$$
(u-z) v+\left(u^{\prime}+\frac{1}{2} \alpha\right)=0 .
$$

Moreover, the transformation (5) becomes

$$
u=v^{\prime \prime \prime}-v v^{\prime \prime}+3\left(v^{\prime}\right)^{2}-4 v^{2} v^{\prime}+v^{4} .
$$

Introducing again a new variable $y(z), y=\frac{1}{u-z}$, Eq. (28) becomes

$$
v=\frac{\left(y^{\prime}+v y^{2}\right)}{y}
$$

where $v=-\frac{1}{2} \alpha-1$, and Eq. (29) becomes

$$
y=\frac{1}{v^{\prime \prime \prime}-v v^{\prime \prime}+3\left(v^{\prime}\right)^{2}-4 v^{2} v^{\prime}+v^{4}-z} .
$$

Substituting $v$ from (30) into (31) yields the following fourth order ODE in $y$ :

$$
y^{(4)}=\frac{5 y^{\prime} y^{\prime \prime \prime}}{y}-5\left[\frac{\left(y^{\prime}\right)^{2}}{y^{2}}-v^{2} y^{2}\right] y^{\prime \prime}-5 v^{2} y\left(y^{\prime}\right)^{2}-v^{4} y^{5}+z y+1 \text {. }
$$


Case 2: $B_{0}=-\frac{3}{2}$. In this case we have $A_{1}=-2, C_{2}=1, D_{4}=\frac{1}{2}$ and therefore the equation $\phi_{0}=0$ becomes

$$
(2 u+z) v-\left(u^{\prime}+\frac{1}{2} \alpha\right)=0
$$

and the transformation (5) reads

$$
u=v^{\prime \prime \prime}+2 v v^{\prime \prime}+\frac{3}{2}\left(v^{\prime}\right)^{2}-v^{2} v^{\prime}-\frac{1}{2} v^{4} .
$$

Let $y=\frac{2}{2 u+z}$. Then Eq. (33) becomes

$$
v=\frac{-\left(y^{\prime}+v y^{2}\right)}{2 y},
$$

where $v=-\frac{1}{2} \alpha+\frac{1}{2}$, and Eq. (34) becomes

$$
y=\frac{1}{v^{\prime \prime \prime}+2 v v^{\prime \prime}+\frac{3}{2}\left(v^{\prime}\right)^{2}-v^{2} v^{\prime}-\frac{1}{2} v^{4}+\frac{1}{2} z} .
$$

Substituting $v$ from (35) into (36) we get

$$
y^{(4)}=\frac{5 y^{\prime} y^{\prime \prime \prime}}{y}+\frac{15\left(y^{\prime \prime}\right)^{2}}{4 y}-\left[\frac{65\left(y^{\prime}\right)^{2}}{4 y^{2}}-\frac{5}{4} v^{2} y^{2}\right] y^{\prime \prime}+\frac{135\left(y^{\prime}\right)^{4}}{16 y^{3}}+\frac{5}{8} v^{2} y\left(y^{\prime}\right)^{2}-\frac{1}{16} v^{4} y^{5}+z y-2 .
$$

Thus we obtain two BTs, namely the pairs of Eqs. (30), (31) and (35), (36), between Eq. (26) and Eqs. (32) and (37), respectively. We now use these results to derive auto-BTs for Eq. (26).

\subsubsection{Auto-Bäcklund transformations for Eq. (26)}

Proceeding analogously as in the case of Eq. (13), and using the fact that Eqs. (32) and (37) are invariant under $v \rightarrow-v$, we obtain the following two auto-Bäcklund transformations for Eq. (26):

$$
\begin{aligned}
& \bar{v}=v+\frac{\alpha+2}{v^{\prime \prime \prime}-v v^{\prime \prime}+3\left(v^{\prime}\right)^{2}-4 v^{2} v^{\prime}+v^{4}-z}, \\
& \bar{\alpha}=-\alpha-4
\end{aligned}
$$

and

$$
\begin{aligned}
& \bar{v}=v-\frac{\alpha-1}{2 v^{\prime \prime \prime}+4 v v^{\prime \prime}+3\left(v^{\prime}\right)^{2}-2 v^{2} v^{\prime}-v^{4}+z}, \\
& \bar{\alpha}=-\alpha+2 .
\end{aligned}
$$

Thus we see, once again, that our approach allows us to derive auto-BTs for the equation under consideration, as well as BTs to other ODEs (the above ODEs in $y$ ). We note that auto-BTs for the similarity reduction of the modified Sawada-Kotera/Kaup-Kupershmidt equation, i.e. (26) have also been given in [43]; these results were later extended to auto-BTs for the entire reduced modified Sawada-Kotera/Kaup-Kupershmidt hierarchy in [44].

\section{Higher order Painlevé equations}

We consider in this section the construction of BTs and auto-BTs for two sixth order Painlevé equations, these being higher order analogues of those considered in Section 2. The procedure is analogous to that outlined in Section 2, and for this reason we do not give all details here.

The two sixth order Painlevé equations we consider here are of the form

$$
v^{(6)}=P\left(z, v, v^{\prime}, \ldots, v^{(4)}\right),
$$

where $P$ is a polynomial in $v, v^{\prime}, \ldots, v^{(4)}$, linear in $v^{(4)}$, with coefficients functions of $z$. We seek a BT of the form

$$
G\left(z, v, v^{\prime}, v^{\prime \prime}, v^{\prime \prime \prime}, v^{(4)}\right) u-\left[v^{(5)}+F\left(z, v, v^{\prime}, v^{\prime \prime}, v^{\prime \prime \prime}, v^{(4)}\right)\right]=0
$$


such that $u$ is the solution of another sixth order Painlevé equation. As in the previous section we use an abbreviated notation and rewrite this $\mathrm{BT}$ as

$$
\begin{aligned}
v^{(5)}= & \left(A_{1} v+A_{0}\right) v^{(4)}+B_{0} v^{\prime} v^{\prime \prime \prime}+\left(C_{2} v^{2}+C_{1} v+C_{0}\right) v^{\prime \prime \prime}+D_{0}\left(v^{\prime \prime}\right)^{2}+\left(E_{1} v+E_{0}\right) v^{\prime} v^{\prime \prime}+\left(F_{3} v^{3}+F_{2} v^{2}+F_{1} v+F_{0}\right) v^{\prime \prime} \\
& +G_{0}\left(v^{\prime}\right)^{3}+\left(H_{2} v^{2}+H_{1} v+H_{0}\right)\left(v^{\prime}\right)^{2}+\left(K_{4} v^{4}+K_{3} v^{3}+K_{2} v^{2}+K_{1} v+K_{0}\right) v^{\prime} \\
& +L_{6} v^{6}+L_{5} v^{5}+L_{4} v^{4}+L_{3} v^{3}+L_{2} v^{2}+L_{1} v+L_{0},
\end{aligned}
$$

where each coefficient $A_{j}, B_{j}, \ldots, L_{j}$ is linear in $u$ with coefficients functions of $z$. Differentiating Eq. (44) and substituting for $v^{(6)}$ from (42) and for $v^{(5)}$ from (44) we obtain an expression linear in $v^{(4)}$, i.e. analogous to the relation (11) obtained in the fourth order case. Elimination of $v$ between this expression and (43) leads to an ODE in $u$.

Here, as in the previous section, we consider a simplification of this elimination procedure: we choose the coefficients $A_{j}, B_{j}, \ldots, L_{j}$ in the BT (44) in order that this expression reduces to a polynomial in $v$. We now turn to our examples.

\subsection{Example 3.1}

As our first example we consider the equation

$$
\begin{aligned}
v^{(6)}= & 14 v^{2} v^{(4)}+56 v v^{\prime} v^{\prime \prime \prime}+42 v\left(v^{\prime \prime}\right)^{2}+70\left(v^{\prime}\right)^{2} v^{\prime \prime}-70 v^{4} v^{\prime \prime}-140 v^{3}\left(v^{\prime}\right)^{2}+20 v^{7} \\
& -\gamma\left(v^{(4)}-10 v^{2} v^{\prime \prime}-10 v\left(v^{\prime}\right)^{2}+6 v^{5}\right)-\beta\left(v^{\prime \prime}-2 v^{3}\right)+z v+\alpha,
\end{aligned}
$$

which is the third member of the generalized $P_{\mathrm{II}}$ hierarchy [42], i.e. it consists of a linear combination of members of the original $P_{\mathrm{II}}$ hierarchy given in [5].

In order that the expression linear in $v^{(4)}$ resulting from the compatibility of our Eq. (45) and the BT (44) reduce to a polynomial in $v$, we have to make the following choice of coefficients: $A_{0}=C_{1}=E_{0}=F_{2}=F_{0}=$ $H_{1}=K_{3}=K_{1}=L_{5}=L_{3}=L_{1}=0, A_{1}=-2 \epsilon, B_{0}=2 \epsilon, C_{2}=10, C_{0}=-\gamma, D_{0}=-\epsilon, E_{1}=40, F_{3}=20 \epsilon, F_{1}=-2 \epsilon \gamma$, $G_{0}=10, H_{2}=10 \epsilon, H_{0}=\epsilon \gamma, K_{4}=-30, K_{2}=6 \gamma, K_{0}=-\beta, L_{6}=-10 \epsilon, L_{4}=3 \epsilon \gamma$ and $L_{2}=-\epsilon \beta$, where $\epsilon= \pm 1$. We can also set, without loss of generality, $L_{0}=u$. The resulting polynomial in $v$ is in fact linear:

$$
(2 \epsilon u+z) v-\left(u^{\prime}-\alpha\right)=0
$$

and defines the inverse of the BT (44) for this choice of coefficients.

Introducing the new variable $y(x)=\frac{2 \epsilon}{2 \epsilon u+z}$ and setting $v=\alpha+\frac{\epsilon}{2}$, Eq. (46) becomes

$$
v=\frac{-\epsilon\left(y^{\prime}+v y^{2}\right)}{2 y}
$$

and (43) becomes

$$
y=1 / \Gamma
$$

where

$$
\begin{aligned}
\Gamma= & v^{(5)}+2 \epsilon v v^{(4)}-\left(2 \epsilon v^{\prime}+10 v^{2}-\gamma\right) v^{\prime \prime \prime}+\epsilon\left(v^{\prime \prime}\right)^{2}-2\left(20 v^{\prime}+10 \epsilon v^{2}-\epsilon \gamma\right) v v^{\prime \prime}-10\left(v^{\prime}\right)^{3}-\epsilon\left(10 v^{2}+\gamma\right)\left(v^{\prime}\right)^{2} \\
& +\left(30 v^{4}-6 \gamma v^{2}+\beta\right) v^{\prime}+10 \epsilon v^{6}-3 \epsilon \gamma v^{4}+\epsilon \beta v^{2}+\frac{\epsilon z}{2} .
\end{aligned}
$$

Thus we obtain the BT (47), (48) between Eq. (45) and the sixth order equation in $y$,

$$
\begin{aligned}
y^{(6)}= & 7 \frac{y^{\prime} y^{(5)}}{y}-\frac{63}{2} \frac{\left(y^{\prime}\right)^{2} y^{(4)}}{y^{2}}+14 \frac{y^{\prime \prime} y^{(4)}}{y}+\left(\frac{7}{2} v^{2} y^{2}-\gamma\right) y^{(4)}+\frac{21}{2} \frac{\left(y^{\prime \prime \prime}\right)^{2}}{y}+\left(\frac{231}{2} \frac{\left(y^{\prime}\right)^{2}}{y}-119 y^{\prime \prime}+5 \gamma y-\frac{7}{2} v^{2} y^{3}\right) \frac{y^{\prime} y^{\prime \prime \prime}}{y^{2}} \\
& -\frac{49}{2} \frac{\left(y^{\prime \prime}\right)^{3}}{y^{2}}+\left(\frac{5}{2} \frac{\gamma}{y}+\frac{7}{4} v^{2} y\right)\left(y^{\prime \prime}\right)^{2}+\frac{973}{4} \frac{\left(y^{\prime}\right)^{2}\left(y^{\prime \prime}\right)^{2}}{y^{3}}+\frac{5}{4}\left(7 v^{2}-10 \frac{\gamma}{y^{2}}\right)\left(y^{\prime}\right)^{2} y^{\prime \prime}+\left(\frac{5}{2} \gamma v^{2} y^{2}-\frac{35}{8} v^{4} y^{4}-\beta\right) y^{\prime \prime} \\
& -\frac{2499}{8} \frac{\left(y^{\prime}\right)^{4} y^{\prime \prime}}{y^{4}}+\frac{1575}{16} \frac{\left(y^{\prime}\right)^{6}}{y^{5}}+\frac{1}{8}\left(45 \frac{\gamma}{y^{3}}-\frac{105}{2} \frac{v^{2}}{y}\right)\left(y^{\prime}\right)^{4}+\frac{1}{2}\left(3 \frac{\beta}{y}-\frac{35}{8} v^{4} y^{3}-\frac{5}{2} v^{2} \gamma y\right)\left(y^{\prime}\right)^{2}+\frac{5}{16} v^{6} y^{7}-\frac{3}{8} v^{4} \gamma y^{5} \\
& +\frac{1}{2} v^{2} \beta y^{3}+z y-2 \epsilon .
\end{aligned}
$$


Moreover, a calculation analogous to that in Section 2.1.1 then provides the auto-BT for Eq. (45),

$$
\begin{aligned}
& \bar{v}=v+\frac{2 \epsilon \alpha+1}{2 \Gamma}, \\
& \bar{\alpha}=-(\alpha+\epsilon),
\end{aligned}
$$

where $\Gamma$ is as given previously. Thus we see that our approach allows the derivation of auto-BTs for Eq. (45). We note that since this equation is a member of the generalized second Painlevé hierarchy, its auto-BTs can also be constructed from those of the standard hierarchy in [5].

\subsection{Example 3.2}

We now consider the equation

$$
\begin{aligned}
v^{(6)}= & -7 v^{\prime} v^{(4)}+7 v^{2} v^{(4)}-14 v^{\prime \prime} v^{\prime \prime \prime}+28 v v^{\prime} v^{\prime \prime \prime}+21 v\left(v^{\prime \prime}\right)^{2}+28\left(v^{\prime}\right)^{2} v^{\prime \prime}+14 v^{2} v^{\prime} v^{\prime \prime}-14 v^{4} v^{\prime \prime} \\
& +\frac{28}{3} v\left(v^{\prime}\right)^{3}-28 v^{3}\left(v^{\prime}\right)^{2}+\frac{4}{3} v^{7}+z v-\frac{\alpha}{2},
\end{aligned}
$$

which corresponds to the similarity reduction of the seventh order member of the modified Sawada-Kotera/KaupKupershmidt hierarchy; see [44]. We proceed as in Section 3.1: in order that the expression linear in $v^{(4)}$ resulting from the compatibility of Eqs. (53) and (44) reduces to a polynomial in $v$, we have to choose $A_{0}=C_{0}=F_{0}=0, B_{0}=-A_{1}-7$, $C_{2}=7-A_{1}^{2}, C_{1}=-A_{1}^{\prime}, D_{0}=\frac{1}{2} A_{1}-\frac{7}{2}, E_{1}=14+3 A_{1}^{2}+7 A_{1}, E_{0}=2 A_{1}^{\prime}, F_{3}=A_{1}^{3}-7 A_{1}, F_{2}=3 A_{1} A_{1}^{\prime}$ and $F_{1}=A_{1}^{\prime \prime}$, and, in addition, we have two possible choices for $A_{1}$, namely $A_{1}=1$ or $A_{1}=-2$. We now consider these two different cases.

Case 1: $A_{1}=1$. In this case we find that $G_{0}=L_{6}=4 / 3, H_{2}=4, K_{4}=-8$ and $H_{1}=H_{0}=K_{3}=K_{2}=K_{1}=$ $K_{0}=L_{5}=L_{4}=L_{3}=L_{2}=L_{1}=0$. We set, without loss of generality, $L_{0}=u$. The resulting polynomial in $v$ is then in fact linear:

$$
(u-z) v+u^{\prime}+\frac{1}{2} \alpha=0 .
$$

Let $y=\frac{1}{u-z}$; then (54) becomes

$$
v=\frac{y^{\prime}+v y^{2}}{y}
$$

where $v=-\frac{1}{2} \alpha-1$, and (43) becomes

$$
y=\frac{1}{\Gamma_{1}},
$$

where $\Gamma_{1}$ is given by

$$
\Gamma_{1}=v^{(5)}-v v^{(4)}+8 v^{\prime} v^{\prime \prime \prime}-6 v^{2} v^{\prime \prime \prime}+3\left(v^{\prime \prime}\right)^{2}-24 v v^{\prime} v^{\prime \prime}+6 v^{3} v^{\prime \prime}-\frac{4}{3}\left(v^{\prime}\right)^{3}-4 v^{2}\left(v^{\prime}\right)^{2}+8 v^{4} v^{\prime}-\frac{4}{3} v^{6}-z .
$$

Thus we obtain the BT (55), (56) between (53) and the sixth order ODE

$$
\begin{aligned}
y^{(6)}= & 7 \frac{y^{\prime} y^{(5)}}{y}+\left(7 \frac{y^{\prime \prime}}{y}-21 \frac{\left(y^{\prime}\right)^{2}}{y^{2}}+7 v^{2} y^{2}\right) y^{(4)}+7 \frac{\left(y^{\prime \prime \prime}\right)^{2}}{y}+\left(42 \frac{\left(y^{\prime}\right)^{3}}{y^{3}}-56 \frac{y^{\prime} y^{\prime \prime}}{y^{2}}-14 v^{2} y y^{\prime}\right) y^{\prime \prime \prime}-\frac{14}{3} \frac{\left(y^{\prime \prime}\right)^{3}}{y^{2}} \\
& +63 \frac{\left(y^{\prime}\right)^{2}\left(y^{\prime \prime}\right)^{2}}{y^{3}}+7 v^{2} y\left(y^{\prime \prime}\right)^{2}-\left(42 \frac{\left(y^{\prime}\right)^{4}}{y^{4}}+14 v^{4} y^{4}\right) y^{\prime \prime}+\frac{4}{3} v^{6} y^{7}+z y+1 .
\end{aligned}
$$

Case 2: $A_{1}=-2$. In this case we have that $G_{0}=16 / 3, \quad K_{4}=-2, \quad H_{2}=10, \quad L_{6}=-2 / 3$ and $H_{1}=H_{0}=K_{3}=K_{2}=K_{1}=K_{0}=L_{5}=L_{4}=L_{3}=L_{2}=L_{1}=0$. We set, again without loss of generality, $L_{0}=u$, and thus obtain the following - again linear-polynomial in $v$,

$$
(2 u+z) v-u^{\prime}-\frac{1}{2} \alpha=0 .
$$


Let $y=\frac{2}{2 u+z}$; then (59) becomes

$$
v=\frac{-\left(y^{\prime}+v y^{2}\right)}{2 y}
$$

where $v=-\frac{1}{2} \alpha+\frac{1}{2}$, and (43) becomes

$$
y=\frac{1}{\Gamma_{2}}
$$

where

$$
\Gamma_{2}=v^{(5)}+2 v v^{(4)}+5 v^{\prime} v^{\prime \prime \prime}-3 v^{2} v^{\prime \prime \prime}+\frac{9}{2}\left(v^{\prime \prime}\right)^{2}-12 v v^{\prime} v^{\prime \prime}-6 v^{3} v^{\prime \prime}-\frac{16}{3}\left(v^{\prime}\right)^{3}-10 v^{2}\left(v^{\prime}\right)^{2}+2 v^{4} v^{\prime}+\frac{2}{3} v^{6}+\frac{1}{2} z .
$$

In this way we obtain the BT (60), (61) between (53) and the sixth order ODE

$$
\begin{aligned}
y^{(6)}= & 7 \frac{y^{\prime} y^{(5)}}{y}+\frac{1}{2}\left(\frac{7}{2} v^{2} y^{2}-\frac{147}{2} \frac{\left(y^{\prime}\right)^{2}}{y^{2}}+35 \frac{y^{\prime \prime}}{y}\right) y^{(4)}+\frac{49}{4} \frac{\left(y^{\prime \prime \prime}\right)^{2}}{y}+\frac{1}{2}\left(\frac{7}{2} v^{2} y y^{\prime}+\frac{609}{2} \frac{\left(y^{\prime}\right)^{3}}{y^{3}}-301 \frac{y^{\prime} y^{\prime \prime}}{y^{2}}\right) y^{\prime \prime \prime} \\
& -\frac{217}{6} \frac{\left(y^{\prime \prime}\right)^{3}}{y^{2}}+\frac{1}{4}\left(1365 \frac{\left(y^{\prime}\right)^{2}}{y^{3}}+7 v^{2} y\right)\left(y^{\prime \prime}\right)^{2}+\frac{1}{8}\left(42 v^{2}\left(y^{\prime}\right)^{2}-7 v^{4} y^{4}-3675 \frac{\left(y^{\prime}\right)^{4}}{y^{4}}\right) y^{\prime \prime} \\
& +\frac{2457}{16} \frac{\left(y^{\prime}\right)^{6}}{y^{5}}-\frac{63}{16} v^{2} \frac{\left(y^{\prime}\right)^{4}}{y}-\frac{21}{16} v^{4} y^{3}\left(y^{\prime}\right)^{2}+\frac{1}{48} v^{6} y^{7}+z y-2
\end{aligned}
$$

In order to get auto-BTs for Eq. (53), we again exploit the fact that our equations in $y$, (58) and (63), are invariant under $v \rightarrow-v$. We thus obtain the following BTs between two solutions $v$ and $\bar{v}$ of Eq. (53), with parameter values $\alpha$ and $\bar{\alpha}$, respectively:

$$
\begin{aligned}
& \bar{v}=v+\frac{\alpha+2}{\Gamma_{1}}, \\
& \bar{\alpha}=-\alpha-4
\end{aligned}
$$

and

$$
\begin{aligned}
& \bar{v}=v-\frac{\alpha-1}{2 \Gamma_{2}}, \\
& \bar{\alpha}=-\alpha+2 .
\end{aligned}
$$

Thus once again we see that our new approach allows the derivation of auto-BTs for the equation under consideration. We note that the above auto-BTs for Eq. (53) were originally obtained in [44]; see also [45]. Also discussed in [44,45] are special integrals of Eq. (53). We note, as is well known, that basic special integrals can be obtained by looking at where auto-BTs, such as (64)-(67), break down. Similarly for the other equations considered in this and the previous section.

\section{Further applications}

In the previous two sections we have considered the application of our algorithm to ODEs of fourth and sixth order. We have seen that in order to recover their auto-BTs, it is sufficient to consider the restricted case whereby the polynomial encountered in $v, v^{\prime}, \ldots, v^{(n-2)}, u, u^{\prime}$ reduces to a polynomial in $v, u, u^{\prime}$. However we have not considered the possibility that we might also be able to recover auto-BTs from a less severely restricted version of our approach. We consider in this section an example of a third order ODE-for which the calculations are therefore sufficiently uncomplicated that it serves as a genuinely illustrative example - for which this is indeed the case.

We take as our example the equation

$$
v^{\prime \prime \prime}=3 \frac{v^{\prime} v^{\prime \prime}}{v}+z v^{\prime}+\alpha v
$$

given in [22]. This equation is related to the second Painlevé equation

$$
y^{\prime \prime}=2 y^{3}+z y+\alpha
$$


via the substitutions

$$
v=\mathrm{e}^{s}, \quad s^{\prime}=y .
$$

Since we know that (69) has auto-BTs, which must then induce auto-BTs for Eq. (68), the question naturally arises of how to recover these last. We note that our interest here is in obtaining auto-BTs for Eq. (68) directly; it may not always be the case that we know how to relate an equation, such as (68), to an ODE for which we know in advance its auto-BTs. Since Eq. (68) has not been considered before, the BTs obtained here are in fact new.

We seek for Eq. (68) a BT of the form

$$
G\left(z, v, v^{\prime}\right) u-\left[v^{\prime \prime}+F\left(z, v, v^{\prime}\right)\right]=0
$$

or equivalently, in our abbreviated notation,

$$
v^{\prime \prime}=\left(A_{1} v+A_{0}\right) v^{\prime}+\left(B_{3} v^{3}+B_{2} v^{2}+B_{1} v+B_{0}\right),
$$

where $A_{j}$ and $B_{j}$ are linear in $u$,

$$
\begin{aligned}
& A_{j}=a_{1 j} u+a_{0 j}, \quad j=0,1, \\
& B_{j}=b_{1 j} u+b_{0 j}, \quad j=0,1,2,3,
\end{aligned}
$$

with coefficients $a_{i j}$ and $b_{i j}$ functions of $z$ only.

Differentiating the BT (72) and using Eq. (68) to replace $v^{\prime \prime \prime}$ and (72) to replace $v^{\prime \prime}$, we obtain

$$
\begin{aligned}
0= & -\left(2 A_{1} v+3 A_{0}\right)\left(v^{\prime}\right)^{2}+\left[A_{1}^{2} v^{3}+\left(2 A_{1} A_{0}+A_{1}^{\prime}-B_{2}\right) v^{2}+\left(A_{0}^{\prime}+A_{0}^{2}-2 B_{1}-z\right) v-3 B_{0}\right] v^{\prime}+A_{1} B_{3} v^{5} \\
& +\left(B_{3}^{\prime}+A_{1} B_{2}+A_{0} B_{3}\right) v^{4}+\left(B_{2}^{\prime}+A_{1} B_{1}+A_{0} B_{2}\right) v^{3}+\left(B_{1}^{\prime}+A_{0} B_{1}+A_{1} B_{0}-\alpha\right) v^{2}+\left(B_{0}^{\prime}+A_{0} B_{0}\right) v
\end{aligned}
$$

We now consider two possibilities.

\subsection{Reducing (75) to a polynomial in $v$}

First of all, proceeding as in our previous examples, we ask that the above equation be polynomial in $v, u, u^{\prime}$; setting equal to zero the coefficients of $\left(v^{\prime}\right)^{2}$ and $v^{\prime}$ requires that $A_{0}=A_{1}=B_{0}=B_{2}=0$ and $B_{1}=-z / 2$. In addition, without loss of generality, we can set $B_{3}=u$. The BT (72) then reads

$$
u=\frac{v^{\prime \prime}+\frac{1}{2} z v}{v^{3}}
$$

and Eq. (75) factors to give

$$
2 u^{\prime} v^{2}-1-2 \alpha=0
$$

which then defines the inverse of (76). Eliminating $v$ between these last two equations then yields the ODE in $u$,

$$
u^{\prime \prime \prime}=\frac{3}{2} \frac{\left(u^{\prime \prime}\right)^{2}}{u^{\prime}}+z u^{\prime}-u(1+2 \alpha) .
$$

This is related to $P_{\text {XXXIV }}$ in [4] by a transformation similar to (70), followed by a further Möbius transformation, with the transformations (76) and (77) then becoming the well-known relations between $P_{\mathrm{II}}$ and $P_{\text {XxxIV. Having obtained }}$ Eq. (78) it should now be possible to recover auto-BTs for Eq. (68), in the same way as in Sections 2 and 3 (see also [8] for $\left.P_{\mathrm{II}}\right)$.

\subsection{Allowing (75) to be linear in $v^{\prime}$}

We now consider the possibility where, instead of reducing (75) to a polynomial in $v, u, u^{\prime}$, we eliminate only the term in $\left(v^{\prime}\right)^{2}$. This then requires only that $A_{0}=A_{1}=0$; then (75) becomes

$$
v^{\prime}=\frac{v\left(B_{0}^{\prime}+B_{1}^{\prime} v+B_{2}^{\prime} v^{2}+B_{3}^{\prime} v^{3}-\alpha v\right)}{3 B_{0}+B_{2} v^{2}+2 B_{1} v+z v}
$$


and the $\mathrm{BT}$ (72) takes the form

$$
v^{\prime \prime}=\left(B_{3} v^{3}+B_{2} v^{2}+B_{1} v+B_{0}\right)
$$

Eliminating derivatives of $v$ between (79) and (80) yields a polynomial in $v$ of degree nine. Since our motivation here is to explore the different ways in which BTs for Eq. (68) can be derived, rather than give a complete analysis, we consider here only the following two possible choices of coefficients:

Case 1: $B_{0}=B_{2}=0$ and $B_{3}=b$ where $b$ is a constant. In this case we can also choose, without loss of generality, $B_{1}=\frac{1}{2}(u-z)$. In this case the BT (72) reads

$$
v^{\prime \prime}=b v^{3}+\frac{1}{2} v(u-z)
$$

and its inverse is defined by

$$
v^{2}=\frac{2 u u^{\prime \prime}-\left(u^{\prime}\right)^{2}-2 u^{3}+2 z u^{2}+(2 \alpha+1)^{2}}{4 b u^{2}},
$$

whereas $u$ satisfies the third order ODE

$$
u^{\prime \prime \prime}=\frac{3 u^{\prime}-2 \alpha-1}{u} u^{\prime \prime}-\frac{3}{2} \frac{\left(u^{\prime}\right)^{3}}{u^{2}}+\frac{(2 \alpha+1)\left(u^{\prime}\right)^{2}}{2 u^{2}}+\frac{2 z u^{2}+3(2 \alpha+1)^{2}}{2 u^{2}} u^{\prime}+2 \alpha u-z(2 \alpha+1)-\frac{(2 \alpha+1)^{3}}{2 u^{2}} .
$$

The relation between this last and $P_{\mathrm{II}}$, as obtained here, would seem to be previously unknown.

Case 2: $B_{0}=B_{3}=0$ and $B_{1}=-\frac{z}{2}$. We choose $B_{2}=u$ without loss of generality, and thus obtain the BT

$$
v^{\prime \prime}=u v^{2}-\frac{1}{2} z v
$$

i.e.

$$
u=\frac{2 v^{\prime \prime}+z v}{2 v^{2}}
$$

with inverse given by

$$
v=\frac{2 u^{\prime \prime}+z u}{2 u^{2}}
$$

The equation satisfied by $u$ is

$$
u^{\prime \prime \prime}=3 \frac{u^{\prime} u^{\prime \prime}}{u}+z u^{\prime}+\bar{\alpha} u,
$$

where $\bar{\alpha}=-\alpha-1$. We therefore conclude that the above BT is in fact an auto-BT for Eq. (68). Thus we see that allowing (75) to be linear in $v^{\prime}$ can also lead to the derivation of auto-BTs for our Eq. (68).

Making the change of variable (70), and similarly for $u$,

$$
u=\mathrm{e}^{t}, \quad t^{\prime}=w,
$$

the BTs (85), (86) become

$$
w=y+\frac{2 \alpha+1}{2 y^{\prime}+2 y^{2}+z} \quad \text { and } \quad y=w+\frac{2 \bar{\alpha}+1}{2 w^{\prime}+2 w^{2}+z}
$$

respectively. The above is of course the well-known BT for $P_{\mathrm{II}}$.

We note in addition that the discrete symmetry $(v, \alpha) \rightarrow(1 / v,-\alpha)$ of Eq. (68)-corresponding to $(y, \alpha) \rightarrow(-y,-\alpha)$ for (69) - can then be used along with the auto-BTs (85) and (86) to obtain the auto-BT

$$
u=\frac{2 v}{4\left(v^{\prime}\right)^{2}-2 v v^{\prime \prime}+z v^{2}}
$$

together with its inverse

$$
v=\frac{2 u}{4\left(u^{\prime}\right)^{2}-2 u u^{\prime \prime}+z u^{2}}
$$

of Eq. (68), where now the parameters $\alpha$ and $\bar{\alpha}$ in (68) and (87) are related by $\bar{\alpha}=-\alpha+1$. 
Finally, it is worth commenting that whilst the auto-BTs (85) and (86) provide mappings between Eqs. (68) and (87) with parameters related via $\alpha+\bar{\alpha}+1=0$, this does not mean that elimination between (85) and (86) yields (68) and (87) in the usual way. Indeed, elimination between (85) and (86) yields two fourth order ODEs whose integration gives (68) and (87) with $\alpha$ and $\bar{\alpha}$ two arbitrary constants of integration; the relation between these parameters is then fixed by insisting that (85) and (86) are auto-BTs for these integrated equations.

However, if to the relations (85) and (86) we add Eq. (79), i.e.

$$
2 u v^{\prime}-2 v u^{\prime}+1+2 \alpha=0,
$$

then these three relations together imply Eqs. (68) and (87) with parameters related by $\alpha+\bar{\alpha}+1=0$, without any need for integration. In this way we have, unusually, a self-consistent triple of equations, whose differential consequence is the two ODEs (68) and (87) with in this last $\bar{\alpha}=-\alpha-1$.

\section{Conclusions}

We have presented a new algorithm to derive BTs for higher order Painlevé equations. We have successfully used this algorithm in order to obtain, amongst other BTs, auto-BTs for a range of ODEs, including for generalized versions of known ODEs and also for ODEs that have not been considered previously. We have also explored the various possible ways that BTs can be obtained using this algorithm.

Generalizations of the approach presented here are of course possible; some possibilities have for example been suggested in [35]. Here we consider further possible generalizations, and also discuss briefly the mathematical foundations underlying this kind of approach to obtaining BTs.

Our main aim here is to clarify a possible misunderstanding of this family of methods of finding BTs. Thus for example, it might be said that the form of Eq. (8), as an equation in $v$, corresponds to an ODE of the polynomial class studied by Chazy [15]. In this way the BT (8) might be referred to as a BT in the polynomial class, and could even be generalized by including against $v^{(3)}$ a coefficient linear in $u, E=e_{1} u+e_{0}$, with either $e_{1}=1$ or $e_{0}=1$.

However, instead of considering BTs in the polynomial class, we could also consider BTs in some non-polynomial class. For example, in the case of third order ODEs, we might seek, instead of (72), a BT of the form

$$
D v^{\prime \prime}=A_{0} \frac{\left(v^{\prime}\right)^{2}}{v}+\left(B_{2} v+B_{1}+B_{0} \frac{1}{v}\right) v^{\prime}+C_{4} v^{3}+C_{3} v^{2}+C_{2} v+C_{1}+C_{0} \frac{1}{v}
$$

where all coefficients are linear in $u$, and with either $D=u+d_{0}$, or $D=d_{1} u+1$. In the same way we could seek, for a fourth order ODE, a BT in the third order non-polynomial class of equation studied in [22].

However, whilst such a classification of BTs is perfectly legitimate - indeed, in later work, we will consider BTs in certain non-polynomial classes - it should not lead to the conclusion that the class of BT considered has to be based on equations which, as equations in $v$, would arise in a Painlevé classification. That is, forms of BT based on non-Painlevé equations can still provide mappings between ODEs with the Painlevé property. This can happen even when considering BTs based on first order equations. For example,

$$
v^{\prime}=v^{3} u
$$

is not a BT in what might be called the Riccati class. However, starting with the ODE

$$
v^{\prime \prime}=6 v^{2},
$$

which has general solution $v=\wp\left(x-x_{0}, 0, g_{3}\right)$, this BT leads to the polynomial in $v$,

$$
3 u^{2} v^{3}+u^{\prime} v-6=0
$$

and thus to the second order third degree ODE in $u$,

$$
9 u^{2}\left(u^{\prime \prime}\right)^{3}-24 u\left(u^{\prime}\right)^{2}\left(u^{\prime \prime}\right)^{2}+16\left(u^{\prime}\right)^{4} u^{\prime \prime}-2916 u^{2} u^{\prime} u^{\prime \prime}+4032 u\left(u^{\prime}\right)^{3}+104976 u^{3}=0 .
$$

The general solution of this last equation is the elliptic function given by $u=\wp^{\prime}\left(x-x_{0}, 0, g_{3}\right) / \wp\left(x-x_{0}, 0, g_{3}\right)^{3}$.

Thus we have two ODEs with the Painlevé property, (95) and (97), such that solutions of (95) are mapped into solutions of (97) via a BT (94) not of some Painlevé class. The inverse of the BT (94) is of course given by (96), or equivalently by the relation $v=\left[27 u u^{\prime \prime}-48\left(u^{\prime}\right)^{2}\right] /\left[6 u u^{\prime} u^{\prime \prime}-8\left(u^{\prime}\right)^{3}-486 u^{2}\right]$. 


\section{Acknowledgements}

The research of PRG and AP is supported in part by the DGICYT under contract BFM2002-02609. PRG and AP thank UM for his invitation to visit Bilkent University, where this work was initiated; they also thank everyone in the Department of Mathematics at Bilkent for their kind hospitality during their stay. PRG thanks the Ministry of Science and Technology of Spain for support under the Programa Ramón y Cajal. AP also acknowledges the support of the Junta de Castilla y León under contract SA011/04.

\section{References}

[1] Painlevé P. Bull Soc Math Fr 1900;28:201-61.

[2] Painlevé P. Acta Math 1902;25:1-85.

[3] Gambier B. Acta Math 1910;33:1-55.

[4] Ince EL. Ordinary differential equations. New York: Dover; 1956.

[5] Airault H. Stud Appl Math 1979;61:31-53.

[6] Boiti M, Pempinelli F. Nuovo Cim B 1980;59:40-58.

[7] Fokas AS, Yortsos YC. Lett Nuovo Cimento 1981;30:539-44.

[8] Fokas AS, Ablowitz MJ. J Math Phys 1982;23:2033-42.

[9] Murata Y. Funkcial Ekvac 1985;28:1-32.

[10] Okamoto K. Ann Mat Pura Appl 1987;146:337-81.

[11] Okamoto K. Jpn J Math 1987;13:47-76.

[12] Okamoto K. Math Ann 1986;275:221-55.

[13] Okamoto K. Funkcial Ekvac 1987;30:305-32.

[14] Gromak V, Laine I, Shimomura S. Painlevé differential equations in the complex plane. Berlin: de Gruyter; 2002.

[15] Chazy J. Acta Math 1911;34:317-85.

[16] Garnier R. Ann Sci École Normale Sup 1912;48:1-126.

[17] Bureau FJ. Ann Mat Pura Appl 1964;66(IV):1-116.

[18] Exton H. Rend Mat 1973;6(6):419-62.

[19] Martynov IP. Differents Uravn 1985;21:764-71, and 937-46.

[20] Muğan U, Jrad F. J Phys A 1999;32:7933-52.

[21] Muğan U, Jrad F. J Nonlinear Math Phys 2002;9:282-310.

[22] Muğan U, Jrad F. Non polynomial third order equations which pass the Painlevé test. Z Naturforsch A 2004;59:163-80.

[23] Cosgrove CM, Scoufis G. Stud Appl Math 1993;88:25-87.

[24] Cosgrove CM. Stud Appl Math 1993;90:119-87.

[25] Cosgrove CM. Stud Appl Math 2000;104:1-65.

[26] Gordoa PR, Pickering A. Europhys Lett 1999;47:21-4.

[27] Gordoa PR, Pickering A. J Math Phys 1999:5749-86.

[28] Gordoa PR, Pickering A. J Phys A 2000;33:557-67.

[29] Gordoa PR, Joshi N, Pickering A. Publ Res Inst Math Sci (Kyoto) 2001;37:327-47.

[30] Sakka A, Muğan U. J Phys A 1997;30:5159-77.

[31] Sakka A, Muğan U. J Phys A 1998;31:2471-90.

[32] Muğan U, Sakka A. J Math Phys 1999;40:3569-87.

[33] Sakka A. J Phys A 2001;34:623-31.

[34] Gordoa PR, Pickering A. Phys Lett A 2001;282:152-6.

[35] Gordoa PR, Pickering A. J Math Phys 2001;42:1697-707.

[36] Gordoa PR. Phys Lett A 2001;287:365-70.

[37] Gordoa PR. Obtaining Bäcklund transformations for higher order ordinary differential equations. In: Gonzalez-Lopez A, Manuel Manas, Martinez Alonso L, Rodriguez MA, editors. Proceedings of the X fall Workshop on Geometry and Physics (Publicaciones de la Real Sociedad Matematica Espanola, vol. 4, Madrid, 2003. p. 255-64).

[38] Sakka A. Phys Lett A 2002;300:228-32.

[39] Gordoa PR, Joshi N, Pickering A. Nonlinearity 1999;12:955-68.

[40] Gordoa PR, Joshi N, Pickering A. Glasgow Math J 2001;43A:23-32.

[41] Gordoa PR, Joshi N, Pickering A. Nonlinearity 2001;14:567-82.

[42] Pickering A. Phys Lett A 2002;301:275-80.

[43] Hone ANW. Physica D 1998;118:1-16.

[44] Joshi N, Pickering A. Bäcklund transformations for similarity reductions of the modified Sawada-Kotera/Kaup-Kupershmidt hierarchy, preprint (1999).

[45] Kudryashov NA. Phys Lett A 2000;273:194-202. 